\title{
Polymorphisms of the BARX1 and ADAMTS17 Locus Genes in Individuals With Gastroesophageal Reflux Disease
}

\author{
Alexandra Argyrou, ${ }^{1}$ Evangelia Legaki, ${ }^{1}$ Christos Koutserimpas, ${ }^{2}$ Maria Gazouli, ${ }^{1 *}$ Ioannis Papaconstantinou, ${ }^{3}$ George Gkiokas, ${ }^{3}$ \\ and George Karamanolis ${ }^{4}$ \\ ${ }^{I}$ Department of Basic Medical Sciences, Laboratory of Biology, School of Medicine, National and Kapodistrian University of Athens, Athens, \\ Greece; ${ }^{2} 2 n d$ Department of General Surgery, "Sismanoglio General Hospital of Athens, Athens, Greece; ${ }^{3}$ 2nd Department of Surgery, School of \\ Medicine, National and Kapodistrian University of Athens, Athens, Greece; and ${ }^{4}$ Gastroenterology Unit, 2nd Department of Surgery, School of \\ Medicine, National and Kapodistrian University of Athens, Athens, Greece
}

\section{Background/Aims}

Gastroesophageal reflux disease (GERD) represents a common condition having a substantial impact on the patients' quality of life, as well as the health system. According to many studies, the BARX1 and ADAMTS17 genes have been suggested as genetic risk loci for the development of GERD and its complications. The purpose of this study is to investigate the potential association between GERD and BARX1 and ADAMTS17 polymorphisms.

\section{Methods}

The present is a prospective cohort study of 160 GERD patients and 180 healthy control subjects of Greek origin, examined for BARX1 and ADAMTS17 polymorphisms (rs11789015 and rs4965272) and a potential correlation to GERD.

\section{Results}

The rs 11789015 AG and GG genotypes were found to be significantly associated with GERD $(P=0.032 ; \mathrm{OR}, 1.65 ; 95 \% \mathrm{Cl}, 1.06$ 2.57 and $P=0.033 ; \mathrm{OR}, 3.00 ; 95 \% \mathrm{Cl}, 1.15-7.82$, respectively), as well as the $\mathrm{G}$ allele $(P=0.007 ; \mathrm{OR}, 1.60 ; 95 \% \mathrm{Cl}, 1.14-2.24)$. Concerning the rs4965272, only the GG genotype was significantly associated with GERD $(P=0.035 ; O R, 3.42 ; 95 \% \mathrm{Cl}, 1.06-11.05)$.

\section{Conclusions}

This is a study investigating the potential correlation between BARX1 and ADAMTS17 polymorphisms and the development of GERD, showing a considerable association between both polymorphisms and the disease. This finding suggests that esophageal differentiation or altered regulation on microfibrils in the cell environment could be implicated as possible mechanisms in the pathogenesis of GERD.

(J Neurogastroenterol Motil 2019;25:436-441)

\section{Key Words}

Gastroesophageal reflux; BARX1; ADAMTS17; Polymorphism, genetic

\footnotetext{
Received: October 30, 2018 Revised: February 1, 2019 Accepted: April 7, 2019

$\circledast$ This is an Open Access article distributed under the terms of the Creative Commons Attribution Non-Commercial License (http://creativecommons. org/licenses/by-nc/4.0) which permits unrestricted non-commercial use, distribution, and reproduction in any medium, provided the original work is properly cited.

${ }^{*}$ Correspondence: Maria Gazouli, PhD Department of Basic Medical Sciences, Laboratory of Biology, School of Medicine, National and Kapodistrian University of Athens, Michalakopoulou 176, 11527 Athens, Greece Tel: +30-2107462231, Fax: +30-2107462231, E-mail: mgazouli@med.uoa.gr
} 


\section{Introduction}

Gastroesophageal reflux disease (GERD) represents a common condition having a substantial impact on the patients' quality of life, as well as the health system. According to the Montreal definition and classification system, GERD develops when the reflux of stomach contents leads to troublesome symptomatology and/ or complications. ${ }^{1}$ Numerous typical symptoms appear in GERD, such as heartburn and acid regurgitation, some atypical ones, like dysphagia and chest pain. Extra-esophageal symptoms, such as chronic cough and laryngitis may also be present. ${ }^{2-4}$ Esophageal exposure to gastric acid in patients with GERD can be severe enough to cause endoscopically visible mucosal damage (erosive esophagitis), esophagus stenosis, and Barrett's esophagus (BE). The latter may subsequently lead to esophageal adenocarcinoma (EAC). A considerable number of patients present no endoscopically visible damage to the esophageal mucosa, despite the symptoms of GERD and esophageal exposure to the acid. This condition has been referred to as non-erosive reflux disease (NERD). ${ }^{5}$

Various factors, such as cellular, molecular, and genetic factors, may affect the severity of GERD. Limited studies have shown that host genetic factors may affect the development of GERD, including genes that can modulate the gastric acid secretion (pre- and anti-inflammatory agents, and those involved in the acid secretion pathway), the DNA repair pathway (for BE and EAC), the carcinogenic pathway for removing toxins (for EAC), cell cycle regulator pathway, and visceral hypersensitivity during acid reflux in the esophagus. ${ }^{6}$ The role of genetics in the pathogenesis of GERD, including $\mathrm{BE}$ and $\mathrm{EAC}$, has been demonstrated by studies on monozygotic and dizygous twins (correlation for gastroesophageal reflux in monozygotic twins), the increased risk of disease in people with a positive family history, and the Genome-Wide Association Studies which recently identified single-nucleotide polymorphisms (SNPs) associated with GERD and its complications. According to many studies, the BARX1 and ADAMTS17 genes have been suggested as genetic risk loci for the development of GERD and its complications. $^{7-10}$

The aim of the study is to evaluate whether BARX1 and $A D$ AMTS17 polymorphisms (rs11789015 and rs4965272, respectively) are associated with gastroesophageal acid reflux risk in our well-defined Greek population. Any negative or positive association of SNPs with GERD represents a new insight in the pathogenesis of the disease.

\section{Materials and Methods}

\section{Study Population}

Our study included 160 GERD patients and 180 sex- and age-matched healthy individuals, all of Greek origin. The patients were recruited at three hospitals, the 2nd Department of Surgery and Gastroenterology unit, School of Medicine, University of Athens; the Department of Gastroenterology, 251 Airforce General Hospital of Athens; and the "Sismanoglio" General Hospital of Athens. Eligible control individuals were adults without symptoms of GERD, and without a history of esophageal or other gastrointestinal cancer, a known diagnosis of BE or reflux esophagitis. In all GERD cases, the diagnosis was endoscopically confirmed (according to the Los Angeles classification for GERD). ${ }^{11}$ Patients who suffered symptoms such as heartburn, chest pain, regurgitation, or dyspepsia for several weeks per month or moderate to severe symptoms more than once per week for at least 3 months were recruited. All patients answered the GERD-Health Related Quality of Life Questionnaire (GERD-HRQL) translated into Greek, for estimating the typical symptoms of GERD. ${ }^{12}$ Exclusion criteria were the presence of gastroenterological surgery, esophageal motility disorders, BE, benign gastric ulcer, duodenal ulcer, gastroduodenal malignancies, and those who were under anti-reflux medication. All participants provided written informed consent before entering into the study. The study was conducted in accordance with the Declaration of Helsinki and was approved by Hospital Ethics committee.

\section{Genotyping}

Genomic DNA was extracted from whole peripheral blood using the Nucleospin Blood Kit (MACHEREY-NAGEL GmbH \& Co. KG, Düren, Germany) according to the manufacturer instructions. Allele specific polymerase chain reaction (PCR) method was used to identify both polymorphisms, rs11789015(A/G) and rs4965272(G/T), where 2 different PCR reactions are performed with one or the other allele specific primer. For the rs 11789015 the primers used were a common forward 5'GGTCCTAAGAAGATGGCGTTTG'3, and a reverse for the G-allele: 5'-GATGGGGAAGCGTCTGAAAAC-3' and one for the A-allele: 5'-GATGGGGAAGCGTCTGAAAAT-3' which produced a 222-bp DNA fragment. For the rs4965272 polymorphism, allele-specific PCR was performed using the following sense primers: rs4965272G 5'- GAGTATATCTTTGTTGCTTCG-3', rs4965272T allele 5'- GAGTATATCTTTGTTGCTTCG-3' 
with the common reverse primer rs4965272R: 5'- ATTCACAGTGTTCCAGGTA-3' which amplified a 229-bp DNA product.

\section{Statistical Methods}

Genotype frequencies were compared with the $\chi^{2}$ test with Yate's correction using S-Plus (v.6.2 Insightful, Seattle, WA, USA). Odds ratios (OR) and 95\% CI were obtained with GraphPad (version 300; GraphPad Software, San Diego, CA, USA). HardyWeinberg equilibrium was verified by calculating the expected frequencies and numbers and was tested separately in patients and controls using the goodness-of-fit $\chi^{2}$ test. The $P$-values are all 2-sided. $P$-values of $<0.05$ were considered significant.

\section{Results}

\section{Study Population and Demographics}

A total of 160 GERD patients, and 180 healthy controls were examined. The demographic characteristics of subjects are presented in Table 1. The study group included 83 males (51.87\%) and 77 females $(48.12 \%)$, with a mean age of $58.05 \pm 24.10$ years. The control group consisted of 92 males $(51.11 \%)$ and 88 females

Table 1. Demographics and Clinical Characteristics of Patients

\begin{tabular}{|c|c|c|c|}
\hline Variables & Controls & GERD & $P$-value (GERD vs controls) \\
\hline Sex & & & 0.888 \\
\hline Male & $92(51.11)$ & $83(51.87)$ & \\
\hline Female & $88(48.89)$ & $77(48.12)$ & \\
\hline Age (yr) & $61.70 \pm 18.50$ & $58.05 \pm 24.10$ & 0.116 \\
\hline Smoking status & & & 0.079 \\
\hline Never & $72(40.00)$ & $66(41.25)$ & \\
\hline Ex-smoker & $76(42.22)$ & $53(33.12)$ & \\
\hline Current & $32(17.78)$ & $41(25.62)$ & \\
\hline $\mathrm{BMI}$ & $25.60 \pm 4.30$ & $27.82 \pm 3.28$ & $<0.001$ \\
\hline Heartburn & & & $<0.001$ \\
\hline Yes & & 146 & \\
\hline No & & 14 & \\
\hline \multicolumn{4}{|l|}{ Chest pain } \\
\hline Yes & & 66 & 0.003 \\
\hline No & & 94 & \\
\hline Acid regurgitation & & & - \\
\hline Never & & 3 & \\
\hline Occasionally & & 40 & \\
\hline Sometimes & & 51 & \\
\hline Frequently & & 42 & \\
\hline Always & & 24 & \\
\hline \multicolumn{4}{|l|}{ Endoscopic findings } \\
\hline \multicolumn{4}{|l|}{ NERD } \\
\hline Yes & & 75 & 0.314 \\
\hline No & & 85 & \\
\hline ERD (LA classification) & & & - \\
\hline A & & 26 & \\
\hline $\mathrm{B}$ & & 37 & \\
\hline $\mathrm{C}$ & & 16 & \\
\hline $\mathrm{D}$ & & 6 & \\
\hline Hiatus hernia & & 76 & \\
\hline Male & & 52 & 0.011 \\
\hline Female & & 24 & \\
\hline
\end{tabular}

GERD, gastroesophageal reflux disease; BMI, body mass index; NERD, non-erosive reflux disease; LA, Los Angeles; ERD, erosive reflux disease. Values are presented as $\mathrm{n}(\%)$ or mean $\pm \mathrm{SD}$. 
$(48.89 \%)$ healthy subjects, with a mean age of $61.70 \pm 18.50$. Gender, age, and smoking status are independent clinical-demographic factors. The mean body mass index in GERD patients was 27.82 \pm 3.28 . The most common symptom, presented in $91.25 \%$ of GERD patients was heartburn, followed by chest pain $(41.25 \%$ of GERD patients). According to the endoscopic findings, as illustrated in Table 1, the GERD cases were graded as A, B, C or D of the classification. Furthermore, in $46.88 \%$ of the patients no visible lesions were found on endoscopy, despite the presence of troublesome reflux-associated symptoms. These patients were characterized as NERD. Hiatus hernia as noticed in Table 1 was founded in $47.50 \%$ of the patients, mainly in male population.

\section{Genotyping Data}

The distribution and frequency of genotypes for cases and controls are shown in Table 2. There were no significant departures from the Hardy-Weinberg equilibrium for either polymorphism in any of the subject groups. Regarding the rs11789015 polymorphism, both AG and GG genotypes frequencies $(P=0.032$ and $P=0.033$ respectively), as well as the minor allele $G(P=$ 0.007 ) were significantly increased in GERD patients compared to healthy controls, exhibiting an association with susceptibility to GERD development. The rs4965272 polymorphism GG genotype was found to be overrepresented in GERD patients compared to healthy controls $(P=0.035)$. On the other hand, it is interesting to note that $\mathrm{TG}$ genotype and the $\mathrm{G}$ allele did not reveal any statistical difference in frequency between the 2 groups $(P=0.540$ and $P=$ $0.068)$.

Table 2. Frequency of rs11789015 and rs4965272 Genotypes and Risk of Gastroesophageal Reflux Disease

\begin{tabular}{lrrl}
\hline Polymorphism & $\begin{array}{c}\text { Controls } \\
(\mathrm{n}=180)\end{array}$ & $\begin{array}{c}\text { GERD } \\
(\mathrm{n}=160)\end{array}$ & $P$-value; OR $(95 \% \mathrm{CI})$ \\
\hline rs11789015 (A/G) & & & \\
AA & 102 & 68 & $1.000[$ reference] \\
AG & 71 & 78 & $0.032 ; 1.65(1.06-2.57)$ \\
GG & 7 & 14 & $0.033 ; 3(1.15-7.82)$ \\
A allele & 275 & 214 & $1.000[$ reference] \\
G allele & 85 & 106 & $0.007 ; 1.603(1.14-2.24)$ \\
rs4965272 (T/G) & & & \\
TT & 128 & 103 & $1.000[$ reference] \\
TG & 48 & 46 & $0.540 ; 1.91(0.74-1.93)$ \\
GG & 4 & 11 & $0.035 ; 3.42(1.06-11.05)$ \\
T allele & 304 & 252 & $1.000[$ reference $]$ \\
G allele & 56 & 68 & $0.068 ; 1.46(0.99-2.16)$ \\
\hline
\end{tabular}

GERD, gastroesophageal reflux disease.

\section{Discussion}

Several studies have suggested that genetic factors substantially explain the pathogenesis of GERD, its complications (BE and EAC) and the phenotypic variance of the severity of some symptoms. ${ }^{13}$ We reported an association between polymorphism of BARX1 (rs11789015) and polymorphism of ADAMTS17 (rs4965272) with the presence of GERD, defined by endoscopic presence or absence of erosive esophagitis. The $B A R X 1$ gene located at the $9 \mathrm{q} 22$ chromosome was suggested by Levine et $\mathrm{al}^{7}$ as susceptible to the development of BE and EAC, whereas rs4965272 polymorphism was indicated by Bonfiglio et $\mathrm{al}^{10}$ meta-analysis as a possible pathogenetic factor for GERD. The functions of the $B A R X$ genes mainly concern gene control and processes involved in myogenic differentiation. Early in gastrointestinal development, expression of the $B A R X 1$ gene is limited to stomach mesenchyme. $B A R X 1$ mainly regulates the expression of $s F R P 1$ and $s F R P 2$, genes that reduce Wnt's (Wingless-related integration site) activity. These signals act on the endoderma to differentiate the stomach epithelium. ${ }^{8,14}$ Especially, the rs 11789015 locus, which is located in intron 3 of the gene homeobox protein BarH-like 1b (BARX1) at the $9 \mathrm{q} 22$ chromosome, encodes a transcription factor important in esophageal differentiation. ${ }^{15}$ It is found that dysregulation of this gene is susceptible to the development of $\mathrm{BE}$ and $\mathrm{EAC}$, probably by reducing anatomical anti-reflux mechanisms. ${ }^{7-9}$ In our study, patients carrying the heterozygous AG genotype or the homozygous GG genotype at the rs11789015 polymorphism of the BARX1 gene demonstrated an increased odds ( $P=0.030$ each) of developing GERD. These findings are in accordance with the studies mentioned correlating the polymorphism with the onset of the complications of GERD.

Furthermore, we showed that carriers of the homozygous GG genotype of the ADAMTS17 polymorphism (rs4965272) have 3.42-fold increased odds of GERD compared to those carrying the TT genotype, while the presence of $\mathrm{G}$ allele alone is not sufficient to increase the odds. The ADAMTS17 gene codes for a member of the ADAMTS (a disintegrin and metalloproteinase with thrombospondin motifs) protein family, which comprises of 19 secreted proteases primarily associated with the extra-cellular matrix and is involved in a wide range of human biological processes with potential roles in arthritis, cancer, angiogenesis, atherosclerosis, central nervous system disorders, and fertility. ${ }^{16,17}$ ADAMTS17 is widely expressed in human tissues but the function of this protein has not been yet determined. Mutations in ADAMTS17 have 
been identified as a cause of autosomal-recessive Weill-Marchesani syndrome. ${ }^{18}$ This gene could be expressed at different levels in different cell types and give rise to different regulatory influences on microfibrils in the cell environment. ${ }^{19}$ From a newly published meta-analysis of reflux genome-wide association, it is revealed that ADAMTS17 and especially its SNP rs4465272, is strongly associated with the GERD pathogenesis. ${ }^{10}$

Acid regurgitation and heartburn were the cardinal symptoms among the GERD patients. Acid regurgitation was described in approximately $98.13 \%$ of the patients, mentioned mostly as a frequent symptom during the week, described as a sour taste in the mouth or as a sense of fluid moving up and down in the chest. Heartburn was described as a burning sensation under the sternum, differentiated with chest pain, the latter defined as angina or a pressure or squeezing pain behind the sternum. Furthermore, it is widely accepted that the incidence of reflux symptoms rises progressively with increasing body mass index. The latter was measured in both controls and GERD patients. A correlation between GERD and overweight patients was found, which is in accordance with previous studies. ${ }^{13}$

There are several limitations to this study. First, the study population is a small sample taken from 3 hospitals and do not necessarily represent the general population. Second, according to the demographic's characteristics, we could not reach conventional levels of statistical significance for gender or smoking status. Recent studies revealed a correlation between the female sex and the onset of GERD, with the female hormones (progesterone and estrogen) and the more frequent existence of hiatal hernia in women as potential risk factors. ${ }^{20-22}$ Moreover, according to smoking, Zheng et $\mathrm{al}^{23}$ concluded that a dose-dependent smoking was linked with the occurrence of GERD. Respectively, the latter could explain the absence of association between smoking and GERD in our study, since we did not measure the quantity of cigarettes, but only collected data whether the patients were current smokers or non-smokers. Third, dietary patterns, physical activity, additional medications affecting esophageal and gastric motility, and other environmental exposures were not accounted. Under these conditions, our results may be attenuated, and our associations may actually be stronger than reported.

In conclusion, this study provides evidence of a correlation between the objective presence of GERD and both the BARX1 (rs11789015) and ADAMTS17 (rs4965272) polymorphisms in a Greek population. The relationship with $B A R X 1$ may be related to its role to the esophageal differentiation. The ADAMTS17 association could be allied with its influence on microfibrils in the cell environment, as its specific role has not yet been determined.
Further investigation in larger population groups and in different nationalities is needed to confirm these findings.

\section{Financial support: None.}

\section{Conflicts of interest: None.}

Author contributions: We certify that all authors have contributed to and agreed on the content of the manuscript. Ioannis Papaconstantinou, George Karamanolis, and George Gkiokas conceived of the presented idea; Maria Gazouli designed the study; Alexandra Argyrou and Evangelia Legaki performed the experiments and wrote the article with support from Maria Gazouli, Ioannis Papaconstantinou, and George Karamanolis; Alexandra Argyrou and Christos Koutserimpas contributed to data collection; Alexandra Argyrou, Evangelia Legaki, and Maria Gazouli contributed to the interpretation of the results; and Maria Gazouli, Ioannis Papaconstantinou, George Gkiokas, and George Karamanolis made the critical revision of the article and the final approval of the version to be published.

\section{References}

1. Vakil N, van Zanten SV, Kahrilas P, Dent J, Jones R; Global Consensus Group. The definition and classification of gastroesophageal reflux disease: a global evidence-based consensus. Am J Gastroenterol 2006;101:1900-1920.

2. De Giorgi F, Palmiero M, Esposito I, Mosca F, Cuomo R. Pathophysiology of gastro-oesophageal reflux disease. Acta Otorhinolaryngol Ital 2006;26:241-246.

3. Galmiche JP, Bruley des Varannes S. Symptoms and disease severity in gastro-oesophageal reflux disease. Scand J Gastroenterol Suppl 1994;201:62-68.

4. Katz PO, Gerson LB, Vela MF. Guidelines for the diagnosis and management of gastroesophageal reflux disease. Am J Gastroenterol 2013;108:308-328.

5. Ghoshal UC, Chourasia D. Gastroesophageal reflux disease and Helicobacter pylori: what may be the relationship? J Neurogastroenterol Motil 2010;16:243-250.

6. Bredenoord AJ. Mechanisms of reflux perception in gastroesophageal reflux disease: a review. Am J Gastroenterol 2012;107:8-15.

7. Levine DM, Ek WE, Zhang R, et al. A genome-wide association study identifies new susceptibility loci for esophageal adenocarcinoma and Barrett's esophagus. Nat Genet 2013;45:1487-1493.

8. Becker J, May A, Gerges C, et al. Supportive evidence for FOXP1, $B A R X 1$, and FOXF1 as genetic risk loci for the development of esophageal adenocarcinoma. Cancer Med 2015;4:1700-1704.

9. Palles C, Findlay JM, Tomlinson I. Common variants confer susceptibility to Barrett's esophagus: insights from the first genome-wide association 
studies. Adv Exp Med Biol 2016;908:265-290.

10. Bonfiglio F, Hysi PG, Ek W, Karhunen V, Rivera NV, Männikkö M, Nordenstedt H. A meta-analysis of reflux genome-wide association studies in 6750 Northern Europeans from the general population. Neurogastroenterol Motil Published Online First: 2016 Aug 3. doi: 10. 1111/ nmo. 12923.

11. Sami SS, Ragunath K. The classification of gastroesophageal reflux disease. Video J Encycl GI Endsc 2013;1:103-104.

12. Velanovich V. The development of the GERD-HRQL symptom severity instrument. Dis Esophagus 2007;20:130-134.

13. Argyrou A, Legaki E, Koutserimpas C, et al. Risk factors for gastroesophageal reflux disease and analysis of genetic contributors. World J Clin Cases 2018;6:176-182.

14. Kim BM, Buchner G, Miletich I, Sharpe PT, Shivdasani RA. The stomach mesenchymal transcription factor Barx1 specifies gastric epithelial identity through inhibition of transient Wnt signaling. Dev Cell 2005;8:611-622

15. Woo J, Miletich I, Kim BM, Sharpe PT, Shivdasani RA. Barx1-mediated inhibition of Wnt signaling in the mouse thoracic foregut controls tracheo-esophageal septation and epithelial differentiation. PLoS One 2011;6:e22493.

16. Kelwick R, Desanlis I, Wheeler GN, Edwards DR. The ADAMTS (A
Disintegrin and Metalloproteinase with Thrombospondin motifs) family. Genome Biol 2015;16:113.

17. Dubail J, Apte SS. Insights on ADAMTS proteases and ADAMTSlike proteins from mammalian genetics. Matrix Biol 2015;44-46:24-37.

18. Morales J, Al-Sharif L, Khalil DS, et al. Homozygous mutations in ADAMTS10 and ADAMTS17 cause lenticular myopia, ectopia lentis, glaucoma, spherophakia, and short stature. Am J Hum Genet 2009;85:558-568.

19. Hubmacher D, Apte SS. Genetic and functional linkage between ADAMTS superfamily proteins and fibrillin-1: a novel mechanism influencing microfibril assembly and function. Cell Mol Life Sci 2011;68:31373148.

20. Fakhre Yaseri H. Gender is a risk factor in patients with gastroesophageal reflux disease. Med J Islam Repub Iran 2017;31:58.

21. Kim YS, Kim N,Kim GH. Sex and gender differences in gastroesophageal reflux disease. J Neurogastroenterol Motil 2016;22:575-588.

22. Ramya RS, Jayanthi N, Alexander PC, Vijaya S, Jayanthi V. Gastroesophageal reflux disease in pregnancy: a longitudinal study. Trop Gastroenterol 2014;35:168-172.

23. Zheng Z, Nordenstedt H, Pedersen NL, Lagergren J, Ye W. Lifestyle factors and risk for symptomatic gastroesophageal reflux in monozygotic twins. Gastroenterology 2007;132:87-95. 